\section{The effect of structure and rate on the recognition and description of auditory temporal patterns*}

\author{
DAVID PREUSSER† \\ Yale University, New Haven, Connecticut 06510
}

Each trial consisted of dichotomous (high and low frequency) tones started in a random sequence which changed to a patterned sequence. $S$ pressed a switch when he recognized that the sequence was patterned and pressed it again when he could describe the pattern. Rate of presentation was varied. Descriptions were verbalized or tapped. The data showed that descriptions were begun with the longest run of identical tones at the slow rate $(1$ tone/sec) and ended with the longest run at the fast rate (4 tones/sec). This effect was greater with verbalized than with tapped descriptions. At the medium rate (2 tones/sec), descriptions began with the longest run for patterns of nine tones or more and ended with the longest run for patterns of eight tones or less. Pattern recognition occurred substantially sooner than pattern description. The difference in time between these two responses remained constant across rate, while the difference in number of tones increased with rate. The results are discussed in terms of mode of processing, level of processing, and short-term storage.

The temporal patterns used in this study were continuously repeating sequences of events. The sequences were of several different lengths, and each event was dichotomous (high vs low tone). When any of these temporal patterns is continuously repeated to form a single ongoing sequence and $S$ is asked to describe this sequence, measures of both pattern difficulty and perceived pattern organization can be determined. Pattern difficulty is measured by how long $S$ must hear the pattern before he can provide a correct description (delay) and how often he describes the pattern incorrectly (errors). Delay and errors have been shown to correlate postively (Royer \& Garner, 1966; Preusser, Garner, \& Gottwald, 1970b), with delay being the more sensitive measure. Perceived organization is measured by examining the point at which $S$ begins his description of the sequence since any temporal pattern, when continuously repeated, may be correctly described as beginning at any point. For instance, the temporal pattern H(high) L(low) H L L which under continuous repetition becomes H L H L L H L H L L H L H ..., can be correctly described as $H \dot{L}, \mathcal{L} L$; L H L L H; H L L H L; etc.

*This research was supported by Grant MH 14229 from the National Institute of Mental Health to. Yale University, and this paper is based upon a doctoral dissertation presented to the faculty of Yale University. The author wishes to thank Dr. W. R. Garner for his guidance throughout this research and Drs. Jay Braun, Robert G. Crowder and Richard L. Gottwald for their thoughtful criticirm. + Now at Dunlap and Associates, 1 Parkland Drive, Darien, Connecticut, 06820
Temporal Pattern Organization

The first study to investigate the perception of these temporal patterns was conducted by Royer and Garner in 1966. Their results for 8-event auditory temporal patterns showed that certain points in the sequence were consistently preferred over other points for use as the beginning of a pattern description. Also, it was reasonably clear that these preferred points were being determined by the structural properties of the pattern. Royer and Garner (1970), using 9-event auditory temporal patterns, found that at least one of these structural properties is the run of identical events. Ss rarely, if ever, begin their response in the middle of a run. Therefore, the pattern H H H H L H L L L... would be described as $\mathrm{HHH}$ H H L L ;

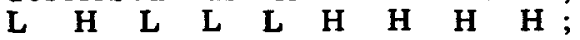
$\begin{array}{llllllllllll}H & L & L & L & H & H & H & H & L & \text { : } & \text { o } & \text { r }\end{array}$ L L L H H H H L H; and not for instance as $\mathrm{H} \mathrm{H} \mathrm{L} \mathrm{H} \mathrm{L} \mathrm{L} \mathrm{L} \mathrm{H} \mathrm{H}$ or L L H H H H LH L. (Vitz, 1968, using learning tasks, has also emphasized the importance of runs of identical events.) This finding makes it possible to code all patterns in terms of run lengths such that the above pattern becomes 4113 , with alternative organizations $4113,1134,1341$, and 3411.

Preusser, Garner, and Gottwald (1970a) found that the particular run chosen to start the description is both a function of figure-ground properties between the two tones and two experimentaly derived organizing principles. They pointed out that any of these temporal patterns can be thought of as the interlocking of two one-element patterns. For example, the interlocking components for 4113 (H H H L H L L) are $\mathrm{HH} \mathrm{H} \mathrm{H}$ where a "- " or gap in the one-element pattern. Their Ss heard and then described a wide range of one-element patterns. From these descriptions there emerged two organizing principles: Gap Principle-Ss tend to end their description with the longest gap (e.g., $\mathbf{H} \mathbf{H} \mathbf{H} \mathbf{H}-\mathbf{H}---$ and L $\quad$ L L L $\quad---$ ); $\begin{array}{lll}\text { and } \\ \text { Run }\end{array}$ Principle-Ss tend to $\bar{b} \overline{\bar{L}} \overline{\mathrm{L}}$ their descriptions with the longest run (e.g., $\mathrm{H}_{\mathrm{H}} \mathbf{H} \mathrm{H}_{-} \mathrm{H}_{-} \ldots$ and $\mathrm{L} \mathbf{L} \mathbf{L}$ - L_).

For some one-element patterns, the two organizing principles predict the same description (e.g., the high e $l$ e $m$ e $t$ of 4113 ; H H H H one-element patterns are said to have compatible organizing principles. Other one-element patterns, however, have the two organizing principles predicting different descriptions. An example would be the one-element pattern formed from the low element of 4113 where the gap principle predicts $L_{-} L \mathrm{~L} L \ldots$ and the r u p pinciple predicts L L L $L$ patterns are said to have incompatible organizing principles.

Descriptions of the whole (or complete) temporal pattern were directly interpretable from these experimentally derived one-element organizing principles. The interpretation rests on the idea that one of the tones, either one, becomes the figure tone, and the one-element organizing principles applicable for this tone completely determine S's whole pattern description. (The figure tone or element for a given $S$ on any trial is operationally defined as the tone with which he begins his description.) If, for instance, the high tone is the figure tone, $\mathbf{S}$ will usually describe the whole pattern as 4113 ( $H$ H H L H L L L), since this description begins with the longest run and ends with the longest gap for that element. If the low tone or element is the figure element he will describe the pattern as either 3411 (L L L H H H H H ), beginning with the longest run of $L$, or as 1134 (L H L L L H H H H), ending with the longest gap. A later study, under somewhat different experimental conditions (Preuser, Garner, \& Gottwald, 1970b), confirmed that these organizing principles can reliably predict temporal pattern descriptions.

Rate of Presentation

Garner and Gottwald (1968) varied 
rate of presentation for 8-vent temporal patterns and concluded that the tvoe of processing $S$ engaged in was different for slow rates as compared with fast rates. At slow rates (less than two events/sec), their results suggested that temporal patterns are "learned," where learning was thought of as an "unintegrated, derived, intellectualized, and active" process. At fast rates (greater than two events/sec), their results suggested that temporal patterns are "perceived," where perceiving was thought of as an "integrated, immediate, compelling, and passive", process (see also Garner \& Gottwald, 1967). Apparently, as rate of presentation increases, $S$ switches from a derived event-by-event form of processing to an integrated or pattern form of processing.

\section{Present Research}

The Garner and Gottwald (1968) experiment, however, was not able to assess differences in pattern organization as a function of rate of presentation. At the fast rates of presentation, Ss followed the organizing principles discussed earlier; but at slow rates of presentation, Ss simply began their descriptions at the point where $E$ began the sequence. Therefore, at slow rates $S$ was not choosing between alternative organizations but merely accepting the initial organization provided by $\mathbf{E}$. If processing is shifting as rate increases, however, pattern organizations should a ls o be changing. Those pattern-organizing principles most appropriate to an event-by-event storage and coding process need not necessarily be most appropriate for integrated pattern processing. For this reason, in the current research the initial organization was obscured by introducing a series of random events before each pattern, preventing $S$ from knowing exactly when the pattern started.

The introduction of random events also allowed the collection of data on another aspect of pattern processing, namely, pattern recognition. Phenomenally, one can hear that these sequences are patterned before being able to describe the pattern. However, this phenomenon could not be studied in previous research since all sequences were patterned. Pattern recognition was tested in the current research by having $S$ indicate when he knew the ongoing sequence had switched from random to pattern.

The remaining two variables in this study were pattern structure and mode of description. Three different types of pattern structure were used. These different structures were chosen so as to vary the relationships between the organizing principles. There were two different modes of description: verbal
Table 1

Compatible-Incompatible Patterns Analyzed in Terms of Strength of Organizing Principles in Incompatible Element*

Run Difference in Incompatible One-Element Patterns

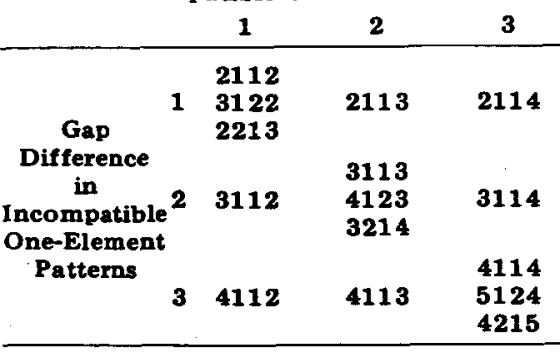

* Strength is the difference between the runs or the gaps in the one-element pattern. $N=15$ patterns.

and tapping. Both have been used in previous research, but as yet no systematic comparison has been conducted.

\section{Purpose}

In summary, the purpose of the present research was to examine the processing of auditory temporal patterns under conditions of varying pattern structure, rate of presentation, mode of description, and criterion of delay in an attempt to better understand the encoding of auditory temporal patterns.

\section{METHOD}

Apparatus

The patterns were punched on paper tape such that one channel of the tape for any pattern contained the pattern and five channels contained unique computer generated random sequences. A tape reader and associated electronic equipment were used to read the tape, switch from a random channel to the pattern channel and produce square-wave tones of 238 $\mathrm{Hz}$ and $275 \mathrm{~Hz}$. Stimulus "on" time equalled blank period between stimuli, and both tones were at $58 \mathrm{~dB}$ sound pressure level.

$S$ sat at a table in a soundproof room. On the table in front of $S$ were two telegraph keys, an intercom (two-way communication with E), a switch, and a speaker.

\section{Task}

For any trial, S's task was to sit back, listen to the sequence of tones, press the switch when he knew that randomness was over and pattern had begun (i.e., the sequence was continuously repeating); then, press the switch again when he could correctly describe the pattern. The second switch press stopped the stimuli. Those Ss who described the pattern verbally were allowed to use any method of description that $E$ could understand. However, they overwhelmingly chose to simply provide run lengths, as "4113." Those Ss who tapped their descriptions were instructed to choose one key, either one, for each tone and tap the appropriate sequence. Each $S$ participated for 44 such trials which took approximately $2 \mathrm{~h}$ to complete. A break was taken halfway through the session.

\section{Experimental Variables}

Patterns. The 22 patterns used in this experiment fall into three general classes: compatible-incompatible, incompatible-incompatible, and gap only-run only. The compatibleincompatible patterns were further subdivided on the basis of "strength of organizing principles" and "length."

Compatible-incompatible patterns are those in which the one-element pattern associated with one of the tones has compatibility with respect to the organizing principles (e.g., $\mathrm{H} \mathrm{H} \mathrm{H} \mathrm{H}-\mathrm{H}--$ of 4113) and the other one-element pattern has incompatibility with respect to the organizing principles (e.g. L L L - - L of 4113). Table 1 shows the actual compatibleincompatible patterns used. It can be seen from Table 1 that these patterns differ with respect to the "strength" of the organizing principles in the incompatible element and with respect to their length.

Strength of the organizing principles is calculated by taking the absolute difference between the runs and the absolute difference between the gaps. For instance, the absolute difference between the lengths of the runs in the incompatible one-element pattern L L L $-\ldots, L-$ is $3-1$, or 2. The absolute difference between the lengths of the gaps is $4-1$, or 3 . Comparing the two, we can say that gap dif $>$ run dif (i.e., $3>2$ ), and previous research has shown that the description predicted by the gap principle would be chosen more often by $S$ than the description predicted by the run principle. The patterns falling on the diagonal in Table 1 all have gap dif = run dif, while the patterns above the diagonal have gap dif $<$ run dif and the patterns below the diagonal have gap dif > run dif in the incompatible one-element pattern. The "strength" of the organizing principles may also be calculated for the compatible element, but this calculation is irrelevant since the principles are not in competition.

Length of a pattern is number of events in the pattern. The shortest pattern, 2111, had only five events while the two longest, 5124 and 5421, had 12 events. Of the compatible-incompatible patterns, eight were "short" ( eight events or 
less) and seven were "long" (nine events or more).

Incompatible-incompatible patterns are patterns in which the one-element pattern associated with each of the tones has incompatibility with respect to the organizing principles. All of these patterns have six runs since such a configuration is impossible to generate with less than six runs. The patterns were: 211211, 311211, 311311 , and 411211. These patterns number only four and are thus too few to allow any further analysis.

Gap only-run only patterns are patterns in which one of the one-element patterns has only the run principle applicable, and the other one-element pattern has only the gap principle applicable. The patterns were: 2111,3111 , and 4111 . If, for instance, the high tone is the figure element in 2111 ( $\mathrm{H} \mathrm{H}-\mathrm{H}$ ), $\mathrm{S}$ has only a run organization, 2111 , since there is no longest gap. In order for $S$ to use a different organization, he must either go counter to the organizing principles, 1121 , or move to the other element, 1112 ( $L_{\ldots} L_{\ldots} \ldots$ ) where the gap principle is applicable.

Rate of presentation. There were three rates of presentation: 1 event/sec (slow), 2 events/sec (medium), and 4 events/sec (fast).

Mode of description. The Ss' pattern descriptions were either tapped on the telegraph keys or given verbally.

Criterion of delay. Two delay criteria were used: pattern recognition and pattern description.

\section{Control Variables}

Four variables were introduced to control for possible artifacts. Three of these-particular random sequence, length of that sequence, and pseudo startpoint-dealt with the random sequence, and the fourth dealt with high vs low tone in the pattern itself.

Each pattern was provided with five unique random sequences. These sequences were generated without constraints, but were later examined on the chance that they could contain an actual repeating pattern. This examination, and the distribution across random sequences of Ss' false alarms (i.e., mistaking randomness for pattern) revealed nothing of significance. Use of these sequences was counterbalanced within each pattern. The number of events heard from any sequence was set at $3,5,7$, or 9 times the number of events in the pattern. These different lengths were counterbalanced within patterns. They were also counterbalanced within Ss so that each $S$ heard an approximately equal number of random events for the entire session. The last control variable dealing with the random sequence was pseudo startpoint. Simply, when the random sequence is over the pattern must begin at some particular point even though $\mathrm{S}$ has no knowledge of this point until he hears the pattern repeat. Therefore, each pattern was begun an equal number of times, insofar as possible, at each event in the pattern. The results indicated that none of these control variables biased the data with respect to any experimental variable, nor did they produce any idiosyncratic effects of their own. Therefore, the use of random sequencing at the beginning of each trial did eliminate the startpoint effect present in previous research without leading to other complications.

Use of the two tones within the pattern was counterbalanced by presenting both the pattern and its complement to each $S$. The complement of a pattern is the pattern with all tones exactly reversed. For instance, the complement of $\mathrm{HHLHL}$ is L L H L H. The results showed that complements, or high vs low tone, did not affect either recognition or description delays differentially. Complements did, however, affect pattern description differentially. First, at the slow rate of presentation, descriptions were begun with the high tone $64 \%$ of the time and the low tone $36 \%$ of the time ( $p<.01$ by sign test). At present, it is not known whether this result is generally true or merely a peculiarity of the specific procedure, tones, etc. There was no difference between the tones at either the medium or fast rate. Second, individual Ss showed clear preferences for consistently beginning their descriptions with one of the two tones. Eighteen Ss began their descriptions with the high tone an average of $86 \%$ of the time while the remaining 12 Ss began with the low tone $88 \%$ of the time (see also Preusser et al., 1970a). These effects were eliminated from overall distributions of pattern descriptions by summing across patterns and complements.

\section{Subjects and Design}

Thirty college students (male and female) from the New Haven, Connecticut, area participated and were paid \$3.50.

Patterns, rate, and criterion of delay were all within-Ss variables. Each $S$ heard each of the 22 patterns and each pattern's complement for a total of 44 trials. Sequencing, or order of presentation for patterns and complements, was counterbalanced across Ss. Rate of presentation for patterns and complements was counterbalanced within Ss and within patterns. All Ss responded with both criteria of delay on all trials.
The only between-Ss variable was mode of description. Half of the Ss (15) gave all of their descriptions verbally and half (15) tapped all of their descriptions. Ss were assigned randomly to each of these two groups.

The entire design, therefore, consisted of 1,320 trials ( 30 Ss by 44 trials). For any single pattern, there were 60 trials (including those trials on which the complement was presented). These 60 trials were divided into six experimental conditions ( 3 rates by 2 modes of description) providing 10 trials for each pattern at each rate with each mode of description.

\section{Data Tabulation}

In summary, each trial provided three measures. First, the delay after the sequence shifted from randomness to pattern until S's first switch press indicated the length of time needed by $S$ to distinguish the patterned sequence from random events. Second, the delay after the sequence shifted until $S$ stopped the sequence with the second switch press indicated the length of time needed by $S$ before he could describe the pattern. Third, S's description of the pattern, verbalized or tapped, indicated which of the available alternative pattern organizations he had used in "learning" or "perceiving" the pattern.

The data for these measures may be summed across patterns as well as across Ss, since all patterns of the same type (compatible-incompatible, incompatible-incompatible, or gap only-run only) are conceptually identical. Each pattern within a given type has the same alternative descriptions when specified in terms of the organizing principles. All of the compatible-incompatible patterns, for instance, have four descriptions which do not break runs of identical events. These descriptions are predicted by, in order, gap and run, gap, neither, and run. Thus the organizations 4113 , 2112, and 5124 are conceptually identical, all being predicted by the gap and run principles; 1134, 1122, and 1245 are all predicted by the gap principle; 1341, 1221, and 2451 are predicted by neither principle; and 3411,2211 , and 4512 are all predicted by the run principle. Summing across patterns within any given type is therefore no more than summing across successive samples of conceptually identical alternative organizations.

\section{RESULTS: DESCRIPTIONS}

As in all previous research in this area, Ss simply did not break runs of identical events when describing a pattern. Of the 1,320 total responses, only 16 broke a run. Of the remaining responses, 186 were errors. These were 
Table 2

Overall Description Data for Compatible-Incompatible Patterns

Rate

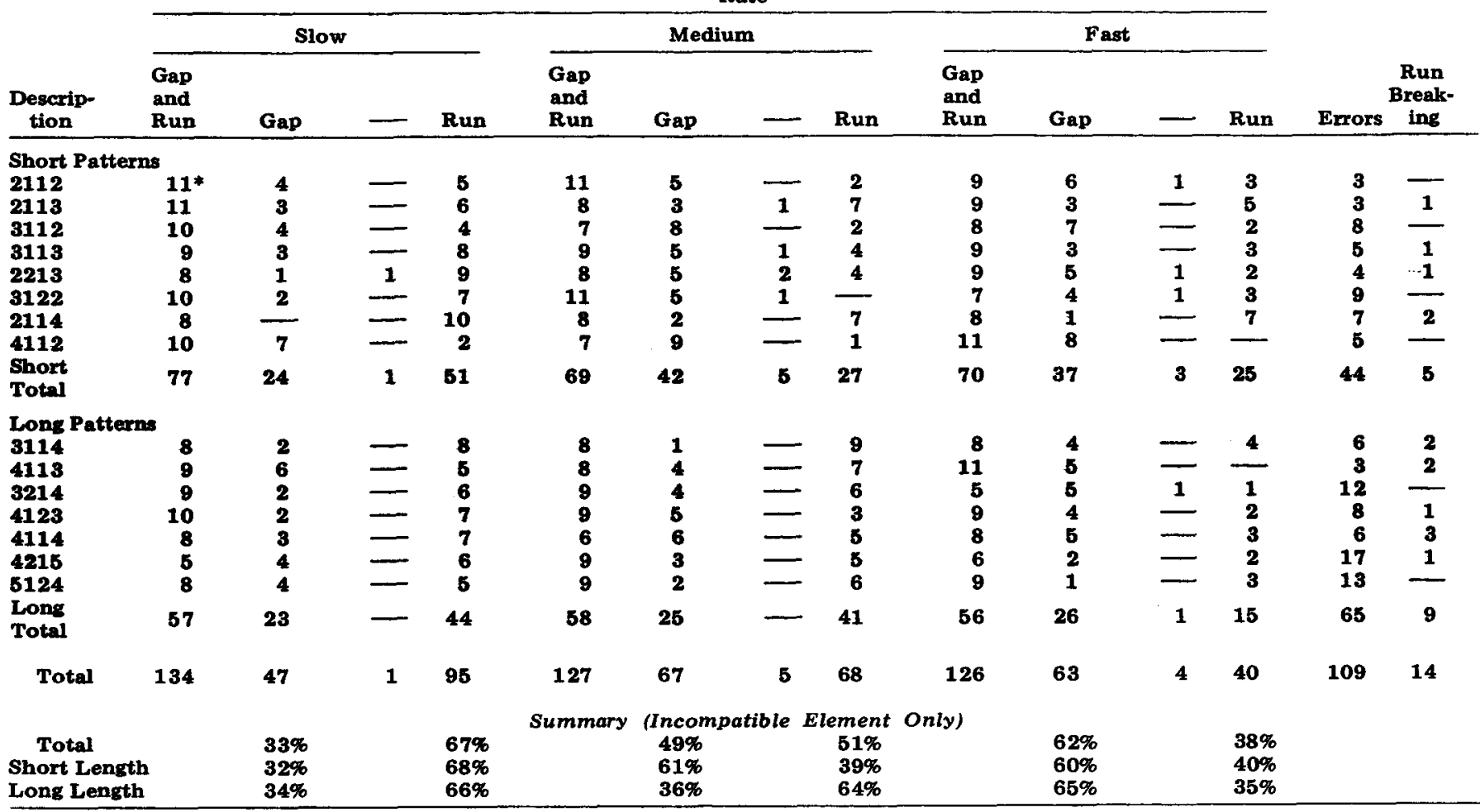

*Entries represent the number of times that pattern was described that way at that rate.

distributed as follows: 29 at the slow rate of presentation, 54 at medium, and 103 at fast. Of the 60 responses/pattern, there was an average of 7,3 errors/pattern for the compatible-incompatible patterns $(9.3$ for the 7 "long" patterns, 5.5 for the 8 "short" patterns), 17.5 errors/pattern for the incompatible-incompatible, and 2.7 errors/pattern for gap only-run only. Errors were evenly distributed across the two modes of description. These data provided no further differential effects. Therefore, the 16 run-breaking descriptions and 186 errors were dropped from further pattern description analyses.

Most of the conclusions from the description data are based on the incompatible element of the compatible-incompatible patterns. The compatible-incompatible patterns were the most widely represented, and the incompatible element is the most interesting since the organizing principles are in opposition. An overview of these data may be seen in Table 2.

\section{Effect of Rate}

The results from the compatible-incompatible patterns clearly showed that pattern descriptions change systematically as a function of rate of presentation. For these patterns, when the incompatible element was the figure element, Ss had a choice between the two organizing principles since one organization is predicted by gap (e.g., 1134) and the other by run (e.g., 3411). At the slow rate of presentation, Ss consistently chose to describe the pattern with the organization predicted by the run principle, while at the fast rate they chose organization predicted by the gap principle. ${ }^{1}$ This effect may be seen in the first summary row of Table 2.

The compatible element of these patterns did not present $S$ with a choice between the organizing principles since one of the two alternative descriptions was predicted by both the gap and run principles (e.g., 4113). The results (averaged across all compatible-incompatible patterns) showed that when the compatible element was the figure element, the description predicted by the gap and run principles was chosen $99 \%$ of the time at the slow rate, $96 \%$ at the medium rate, and $97 \%$ at the fast rate. In other words, when one of the alternative descriptions was predicted by both organizing principles, this description was nearly always chosen regardless of rate of presentation.

The incompatible-incompatible patterns presented $S$ with a choice between the organizing principles, regardless of which element was figure. The results, averaged across both elements for the four patterns of this type showed that use of the description predicted by the gap principle (e.g., 112112) increased from $45 \%$ at the slow rate to $67 \%$ at the medium rate to $86 \%$ at the fast rate. The third alternative description (e.g.,121121), available in these patterns only, was predicted by neither principle and was used less than $4 \%$ of the time at all rates.

Descriptions of the gap only-run only patterns also showed systematic changes as a function of rate. However, these results are directly dependent upon the particular mode of description and will be presented in a later section.

\section{Pattern Variables}

Pattern length. For the 15 compatible-incompatible patterns, eight had eight events or less (short) and seven had nine events or more (long). The secondary summary row of Table 2 shows the data for the incompatible elements of the compatible-incompatible patterns separated by pattern length. It can be seen from Table 2 that pattern length is irrelevant at both slow and fast rates, but critical at the medium rate. 
Table 3

Percent Usage of the Gap and Run Principles for the Incompatible Element of the Compatible-Incompatible Patterns as a Function of Rate and Strength of Organizing Principles

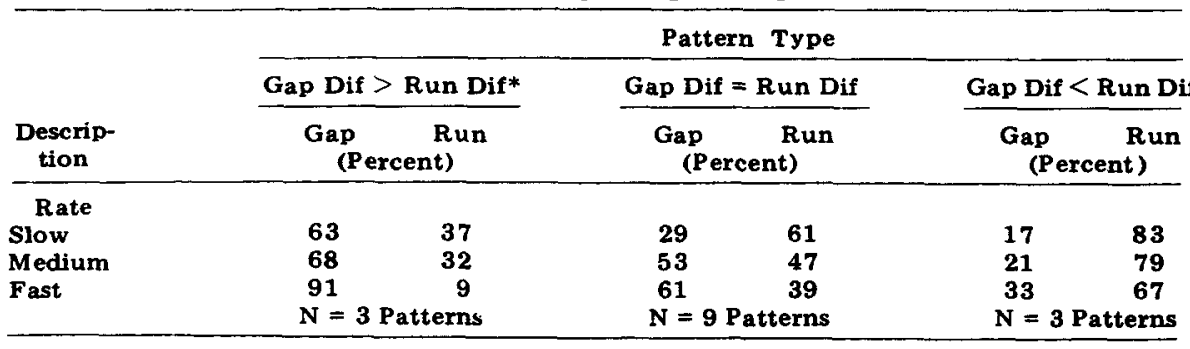

*The specific patterns satisfying each of these conditions are shown in Table 1 .

At that rate, the short patterns are organized by gap and the long patterns are organized by run. In slightly different terms, the distribution of pattern organizations for the short patterns at the medium rate was similar to the distribution for all patterns at the fast rate, while the distribution for the long patterns at the medium rate was similar to all patterns at the slow rate.

Strength of organizing principles. The summary data in Table 2 are averaged across all incompatible elements of the compatibleincompatible patterns. However, it is possible to analyze further the results from these patterns with respect to the relative "strength" of the two organizing principles. Table 3 shows that when the absolute difference between the lengths of the gaps was greater than the absolute difference between the lengths of the runs, the description predicted by the gap principle was consistently favored. Nevertheless, its use increased steadily with rate. When the gap difference equalled the run difference, the relationship was the same as in the first summary row of Table 2. Finally, when the gap difference was less than the run difference, the description predicted by the run principle was consistently chosen. Yet, use of the description predicted by the gap principle still increased steadily with rate. Thus, structural properties of the pattern and rate of presentation were both determining pattern organization.

\section{Mode of Description}

The data for the incompatible element of the compatibleincompatible patterns showed that those Ss who responded verbally increased their use of the description predicted by the gap principle from $29 \%$ at the slow rate to $76 \%$ at the fast rate. However, those Ss who tapped their description only showed an increase of from $37 \%$ to $48 \%$. In other words, the verbal responders were more sensitive to the rate effect than the tapping responders. The results for the incompatible-incompatible patterns also showed the same relationship.

The more interesting verbal vs tapping effects, however, can be seen with the gap only-run only patterns. Here, the two modes of description do not show the same directionality with respect to the effect of rate on descriptions. The gap only-run only patterns ( $N=3$ patterns) have the gap principle predicting one of two possible organizations in one element (e.g., $\mathrm{H}_{-} \mathrm{H}_{-}$) and the run principle predicting one of two possible organizations in the other element (e.g., L L - L _ ). Therefore, the only way Ss could use a particular organizing principle was to use the element where that principle was applicable. The results from these patterns, averaged across $S s$ and patterns, and separated by mode of description, are shown in Table 4. It can be seen from Table 4 that the tappers actually used the description predicted by the gap principle more often at the slow rate than at the fast rate. Though this effect is far from reaching statistical significance, it is important to note that it is even in the opposite direction. The verbal responders, however, clearly showed the rate effect observed with the other types of patterns. Their use of the description predicted by the gap principle increased from $46 \%$ at the slow rate to $81 \%$ at the fast rate.

\section{RESULTS: DELAYS}

Data were collected on two delay criteria. The first was termed "pattern recognition" and was measured by the number of events $S$ heard from the point at which the sequence actually changed from random to pattern until he pressed the switch indicating that he was aware of the change. The second criterion, delay to pattern description, was the number of events from the change until $S$ stopped the sequence (i.e., the second switch press). Across all Ss and patterns, there was never a response for which the second switch press came before the change to pattern. There were, however, 56 instances in which the first switch press came before the sequence actually was a repeating pattern. These responses were distributed by rate: 38 slow, 11 medium, and 7 fast; and by mode of description: 14 verbal and 42 tapping. The trials in which these responses occurred were dropped from the delay analyses. Further, the conclusions were the same for all three types of patterns. Therefore, the results reported here will be for the compatible-incompatible patterns only.

The data showed that as rate of presentation increased, overall delay, as measured by number of events, also increased (See Fig. 1). Figure 1 also shows that delay to pattern description increased more with rate than delay to pattern recognition. Both of these effects are statistically reliable ( $p<.001$ by $F$ test for both). However, there is no a priori justification for measuring delay in terms of number of events. Figure 2 shows the same delays in terms of time. It can be seen in Fig. 2 that as rate of presentation increases, time for both pattern recognition and pattern description decreases, ( $p<.001$ by $F$ test). Further, when delays are in terms of time, there is no Delay Criterion by Rate interaction. The increase in number of events and decrease in number of seconds with increasing rate of presentation is consistent with the findings of Garner and Gottwald (1968). There were no reliable effects associated with mode of description or Ss' usage of alternative pattern organizations.

\section{DISCUSSION}

The most important result of this study was that Ss did organize the same pattern differently as a function of rate of presentation. At the slow rate of presentation, the description predicted by the run principle was used most often, while at the fast rate, the description predicted by the gap principle was used most often. It is felt

Table 4

Percent $U$ ange of the Gap and Run Principles in Descriptions of the Gap Only-Run Only Patterns as a Function of Rate and Mode of Description

\begin{tabular}{|c|c|c|c|c|}
\hline \multirow[b]{3}{*}{$\begin{array}{l}\text { Descrip- } \\
\text { tion }\end{array}$} & \multicolumn{4}{|c|}{ Mode of Description } \\
\hline & \multicolumn{2}{|c|}{ Verbal } & \multicolumn{2}{|c|}{ Tapping } \\
\hline & $\begin{array}{l}\text { Gap } \\
\text { (Per }\end{array}$ & $\begin{array}{l}\text { Run } \\
\text { ent) }\end{array}$ & $\begin{array}{r}\text { Gap } \\
\mathbf{P e}\end{array}$ & Run \\
\hline $\begin{array}{l}\text { Rate* } \\
\text { Slow } \\
\text { Medium } \\
\text { Fast }\end{array}$ & $\begin{array}{l}46 \\
54 \\
81\end{array}$ & $\begin{array}{l}54 \\
46 \\
19\end{array}$ & $\begin{array}{l}68 \\
52 \\
52\end{array}$ & $\begin{array}{l}32 \\
48 \\
48\end{array}$ \\
\hline
\end{tabular}

*Two of the four possible descriptions in these patterns are predicted by neither principle. However, these descriptions accounted for only $6 \%$ of the data and are not included in this analysis. 


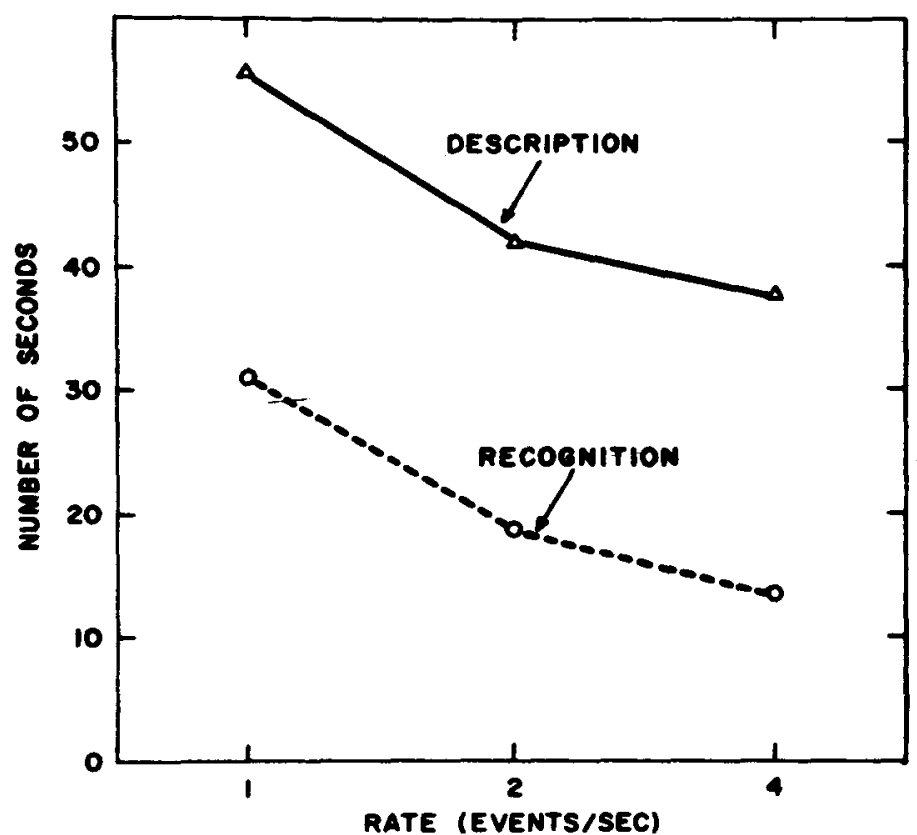

Fig. 1. Mean delay as a function of rate as measured by number of events for both recognizing and describing patterns.

that this effect arises from the fact that the run principle is essentially a "counting" or "event-by-event" principle, while the gap principle is essentially a "timing" principle.

Regardless of rate of presentation, $S$ had to separate each of the successive presentations of the pattern from the ongoing sequence before he could encode the pattern. Only in this way could he distinguish between new events which had to be encoded, and repetitions of old events which he had already begun to encode. The run principle separates each successive presentation of the pattern by "marking" or "cueing" its beginning with the longest run of one of the two tones. When using the run principle, $S$ only needed to select one tone as his figure tone, count the number of events in each run of this tone, determine which run was the longest, and from then on whenever he heard that longest run again he knew the pattern was beginning another repetition. The point, however, is that the longest run can be determined with only counting, or event-by-event information, and little or no information concerning the pattern configuration.

The gap principle, however, appeared to behave in quite a different manner. As before, $\mathbf{S}$ first selected his figure tone; but when using this principle, he separated all of the runs of the figure tone from the ongoing sequence by beginning with that run of the figure tone which organized the pattern with the longest "gap," or run of the ground tone at the end. Placing the longest "gap" at the end meant that the runs of the figure tone were as close together, in time, as was possible and were as distant as possible from their successive repetitions. Thus, if this interpretation is correct, the gap principle does depend on the entire pattern configuration and can be considered as most appropriate for integrated or patterned type of processing. In terms of the learning vs perception distinction suggested by Gamer and Gottwald (1968), the run principle does appear to be most appropriate at the slow rates of presentation (i.e., pattern "learning") and the gap principle appears to be most appropriate at the fast rates (i.e., pattern "perception").

Possible confirmatory evidence for this view of the rate effect on pattern organization is presented by Shepard (1963). He analyzed the data from several experiments investigating the learning of Morse code. The results suggested that during the early stages of learning, and thus with slow rates of presentation, the number of dots and dashes were very important in distinguishing one character from another. However, at later stages of training, and thus faster rates of presentation, the characters which differed only in the number of dots or dashes, and not their overall configuration, were confused the most often. Stage of training and rate of presentation are, of course, confounded in this analysis, but the fact that the number of dots and dashes, regardless of their configuration, is an important distinguishing characteristic at slow rates and not at fast rates is clearly consistent with the present results.

\section{Short-Term Storage}

One of the effects observed in this experiment was that the "short" patterns at the medium rate were described with the organization predicted by the gap principle, while the "long" patterns were described with the organization predicted by the run principle. If the previous analysis concerning the manner in which these two principles operate is correct, we may assume that the short patterns underwent integrated processing and the long patterns underwent event-by-event processing. It is felt that this change in mode of processing as a function of pattern length as well as the change with rate of presentation can be accounted for by postulating a bounded short-term storage or holding mechanism such as the Precategorical Acoustic Store proposed by Crowder and Morton (1969).

The Precategorical Acoustic Store accepts auditory information, and holds this information in an essentilly undifferentiated state for roughly 2-4 sec. Higher processing mechanisms have access to this information for as long as it remains in storage. The 2-4 sec limit on this system dictates that at the slow rate of presentation, only a portion of any long or short pattern could exist in this store at any one time. Therefore, $\mathbf{S}$ could not process more than a few events simultaneously. At the fast rate, 
however, all of the events of any long or short pattern could exist in the store at one time and could be processed simultaneously. The slow rate would then necessitate some form of event-by-event processing while the fast rate allows for integrated processing. At the medium rate, the form of processing which may be engaged in is a function of the length of the pattern. The short patterns can exist in the store in complete form ( 8 events or less at 2 events/sec $=4 \mathrm{sec}$ or less/patterm), while the long patterns cannot $(9$ events or more at 2 events/sec $=4.5 \mathrm{sec}$ or more/pattern).

The short-term storage argument, as presented, does account for the basic effect of rate on auditory pattern organization. However, it assumes two unsettled and important issues. First, the time limits on this storage capacity may be much closer to 2 sec than the 4 sec required by this analysis (see e.g., Crowder, 1969). Second, this argument depends upon the "decay" of information over time and not the "bumping" of old information by new information. Both of these issues must be resolved before the short-term storage explanation can be accepted.

\section{Level of Encoding}

Another factor interacting with rate of presentation to determine pattern organization was mode of description. While conceputally there seems to be little difference between $a$ verbal response and a tapping response, in practice the two modes of description were quite different. The verbal responders normally described the pattern coded in terms of run lengths, as 4113 , while the tappers had to describe the pattern by tapping each discrete event. For this reason, it is felt that the final processing engaged in by the verbal responders before giving their description was further removed from the actual stimulus events than the final processing engaged in by the tappers. It follows, then, that the verbal responders were less bound by the actual stimulus events, and more free to combine, recode, or otherwise process the available information.

The current results can therefore be explained in terms of the greater opportunity or greater freedom afforded the verbal responders to "find" the most appropriate organization. For the compatible-incompatible patterns, they "found" and used the organization predicted by the run principle more often that the tappers at the slow rate and the organization predicted by the gap principle more often than the tappers at the fast rate. The gap only-run only patterns showed this effect even more dramatically. Here, a change in the use of the two principles involves changes in the figure element. The tappers accepted the organization available in their original figure element, while the verbal responders "found" the most appropriate organization regardless of element. Thus, the descriptions of these patterns by the verbal responders showed the rate effect while the descriptions by the tappers did not.

\section{Recomition vs Description}

The most interesting result from the delay data was simply that $\mathrm{Ss}$ can generate a response for pattern recognition which is distinct from the previously used delay to pattern description. Averaged across rate, S recognized that the sequence was patterned a full $23.9 \mathrm{sec}$ before they could describe the pattern while only mistaking the preceding random sequence for pattern $4 \%$ of the time. Still to be determined, however, is whether pattern recognition and pattern description are different levels of the same process or fundamentally different processes.

\section{Theoretical Base}

The conceptual framework of this paper rests primarily on the view that temporal pattern organization can be thought of as a figure-ground phenomenon. The two organizing principles, themselves, are meaningful only if one of the two tones becomes "figure" for $S$ and determines his pattern organization. Therefore, a summary of the support for this view and a consideration of alternative positions would seem appropriate at this time.

In somewhat oversimplified terms, the existing theories of sequential pattern encoding seem to divide themselves into two types; those relying on subunits of the pattern, and those relying on the whole pattern. The impetus for the "subunit" view seems to lie in Miller's (1956) formulations concerning "chunking" of information. Essentially, several "bits" of information can be reduced to a smaller number of "chunks" of information and in this way stored and later retrieved more easily. Restle and Brown (1970) suggested that their $\mathrm{Ss}$ reduced the several events in a sequential pattern to a succession of subunits or a succession of small groups of events. Each subunit reflected some abstract property and this abstract property or rule could later be used to recall or generate this subunit's individual events. Two such abstract properties were "runs" that denote regular progression, as the digits 2-3-4, and "trills" which denote alternation, as 2-3-2. A more complex but essentially similar proposal was presented by Vitz and Todd (1969). Their model starts by grouping individual events into subunits, but now these subunits may be further grouped into increasingly complex units until the entire pattern is encoded. The important characteristic, however, of these models and others that could have been cited is that $S$ starts with individual events and proceeds to "chunk" them together until he has encoded the entire pattern.

The second approach to sequential pattern processing starts with properties of the entire pattern, and not properties of individual events or subunits. The impetus for this approach probably lies in Gestalt psychology, but the modern impetus was provided by Royer and Garner (1966). They found that Ss' descriptions of auditory temporal patterns could not be accounted for by predictions from individual events. The clearest statement of this view, however, can be found in Royer and Garner (1970). They were interested in the organization of temporal patterns and concluded that ". . it is eminently clear from the present data that these temporal patterns are perceived in a wholistic way, with all parts contributing to the nature and acceptability of the particular organization [p. 118]." In other words, $S$ uses properties of the entire pattern to establish how the individual events will be encoded. The figure-ground conception is seen as a direct outgrowth of this point of view. Esentially, figure-ground adds the notion that when $S$ hears the entire pattern he hears one tone, either one, as the figure against the background of the other tone. Since the structure of the figure tone is provided only from its relationship to the ground tone, this is very much a wholistic interpretation. Interestingly, even at the slow rates of presentation, where the information underwent event-by-event processing, $S$ still appeared to be basing his responses on properties of the entire pattern, and organizing principles derived from the figure-ground view predicted organization very well.

Of course, the primary value of any theoretical position lies in its ability to predict and explain the data. None of the data from the present research left any indication that the figure-ground concepts need be replaced or modifed to account for processing of auditory temporal patterns.

\section{REFERENCES}

CROWDER, R. G. Improved recall for digits with a delayed recall cue. Papex presented at Psychonomic Society Meetings, St. Louis, November 6, 1969.

CROWDER, R. G., \& MORTON, J. Precatagorial acoustic storage (PAS). Perception \& Psychophysics, 1969, 5, 365-373. 
GARNER, W. R., GOTTWALD, R. L Some perceptual factors in the learning of sequential patterns of binary events. Journal of Verbal Learning \& Verbal Behavior, 1967, 6, 582-589.

GARNER, W. R., \& GOTTWALD, R. L The perception and learning of temporal patterns. Quarterly Journal of Experimental Psychology, 1968, 20. 97-109.

MILLER, G. A. The magical number seven, plus or minus two: Some limits on our capacity for processing information. Psvcholodical Review. 1956. 63. 81-97. PREUSSER, $\mathbf{D}_{\text {, GARNER, W. R., }}$ GOTTWALD, R. L. Perceptua organization of two-element temporal patterns as a function of their component one-element patterns. American Journal of Psychology, 1970a, 83, 151-170.
PREUSSER, R., GARNER, W. R, \& GOTTWALD, R. L. The effect of starting pattem on descriptions of perceived temporal patterns. Psychonomic Science, $1970 b, 21,219-220$.

RESTLE, F. BROWN, E. R. Serial pattern learning. Journal of Experimental Psychology, 1970, 83, 120-125.

ROYER, F. L., \& GARNER, W. $R$ Response uncertainty and perceptual difficulty of auditory temporal patterns Perception \& Psychophysics, 1966, 1 . 41-47.

ROYER, F. L., \& GARNER, W. $R$ Perceptual organization of nine-element auditory temporal patterns. Perception \& Psychophysics, 1970, 7, 116-120.

SHEPARD, R. N. Analysis of proximities as a technique for the study of information processing in man. Human Factors, 1963,
5, 33-48.

VITZ, P. C. Information, run structure and binary pattern complexity. Perception \& Psychophysics, 1968, 3, 275-280.

VITZ, P. C., \& TODD, T. C. A coded element model of the perceptual processing of sequential stimuli. Psychological Review, 1969, 76, 433-449. NOTE

1. All effects reported involving organization data are significant at least at the .05 level by the sign test or Mann-Whitney $U$ test, where appropriate. Statistics are not reported, however, since the validity of any single result presented here rests more on its relation to the total pattern of results than on its own statistical significance.

(Received for publication March 15, 1971) 